\title{
Erratum to: Segregating gas from melt: an experimental study of the Ostwald ripening of vapor bubbles in magmas
}

Nicole C. Lautze - Thomas W. Sisson •

Margaret T. Mangan - Timothy L. Grove

Published online: 5 August 2010

(C) Springer-Verlag 2010

Erratum to: Contrib Mineral Petrol

DOI 10.1007/s00410-010-0535-x

Due to a typesetting error, equation 1 was printed incorrectly. The equation should read:

$<r>^{3}=t K$

The online version of the original article can be found under doi:10.1007/s00410-010-0535-x.

N. C. Lautze $(\bowtie)$

Istituto Nazionale di Geofisica e Volcanologia-Roma,

Rome, Italy

e-mail: nicole.lautze@ingv.it

N. C. Lautze - T. W. Sisson - M. T. Mangan

U.S. Geological Survey, Volcano Hazards Team,

Menlo Park, CA, USA

T. L. Grove

Massachusetts Institute of Technology,

Cambridge, MA, USA 